УДК 004.738:006.354

\title{
ФУНКЦИОНАЛЬНОЕ И СЕТЕВОЕ ПРЕДСТАВЛЕНИЕ ТИПОВОЙ АРХИТЕКТУРЫ ЦИФРОВОГО ДВОЙНИКА ПРОМЫШЛЕННОГО ПРОИЗВОДСТВА
}

\author{
Андрианова Людмила Прокопьевна \\ д-р техн. наук, профессор \\ Голубев Дэнис Михайлович \\ Уфимский государственный нефтяной технический университет
}

\begin{abstract}
Аннотация: В статье приведены результаты анализа функционального и сетевого представления архитектуры цифрового двойника производства на основе типовой архитектуры промышленного интернета вещей. Приведено функциональное представление архитектуры цифрового двойника производства с декомпозицией на функциональные сущности с описанием их целей и задач. Приведено сетевое представление архитектуры цифрового двойника производства с рассмотрением трех сетей передачи данных: пользовательская сеть, сервисная сеть, сеть передачи данных; для каждой сети дано описание выполняемых функций и технологий передачи данных. Результаты анализа систематизированы и представлены в табличном и графическом виде.

Ключевые слова: цифровой двойник производства, промышленный интернет вещей, структура цифрового двойника производства, типовая архитектура, функциональное представление, сетевое представление.
\end{abstract}

\section{FUNCTIONAL AND NETWORK REPRESENTATION TYPICAL DIGITAL TWIN ARCHITECTURE INDUSTRIAL PRODUCTION}

Andrianova Lyudmila Prokopyevna Doctor of Technical Sciences, Professor Golubev Denis Mikhailovich

\footnotetext{
Abstract: The article presents the results of the analysis of the functional and network representation of the digital twin architecture of production based on the typical architecture of the industrial Internet of Things. The functional representation
} 
of the architecture of the digital twin of production with the decomposition into functional entities with a description of their goals and objectives is given. A network representation of the architecture of the digital twin of production is given with consideration of three data transmission networks: the user network, the service network, and the data transmission network; for each network, a description of the functions performed and data transmission technologies is given. The results of the analysis are systematized and presented in tabular and graphical form.

Key words: digital production twin, industrial internet of things, digital production twin structure, sample architecture, functional representation, network representation.

В условиях перехода России к цифровой экономике промышленное производство трансформируется в умное (интеллектуальное) производство за счет появления цифровых двойников, способных проводить анализ больших данных об объектах, системах или технологических процессах производства еще на стадии нового строительства или реконструкции предприятия [1].

Для сбора и управления большими данными в качестве концептуальной основы цифрового двойника производства используется Интернет вещей (ИВ).

В предварительном национальном стандарте Российской Федерации ПНСТ - 420 «Информационные технологии. Интернет вещей промышленный. Типовая архитектура» определена типовая модель ИВ, включая типовую модель на основе доменов и типовую модели на основе сущностей.

Функциональное представление типовой архитектуры цифрового двойника, отвечающей требованиям ПНСТ - 420 показано на рисунке 1.

Основу функциональной сущности физического производства (PME) составляет сущность FЕ определенного вида ресурсов, т.е. ресурс физического производства конкретных функциональных сущностей, которая может поддерживать различные функции в соответствии с его возможностями [2, с. 8].

Функциональная сущность в DCCE (сущность сбора данных и управления устройствами. Задачи, выполняемые функциональными сущностями сбора данных, предобработки данных, управления, запуска и маркировки, приведены в таблице 1 . 


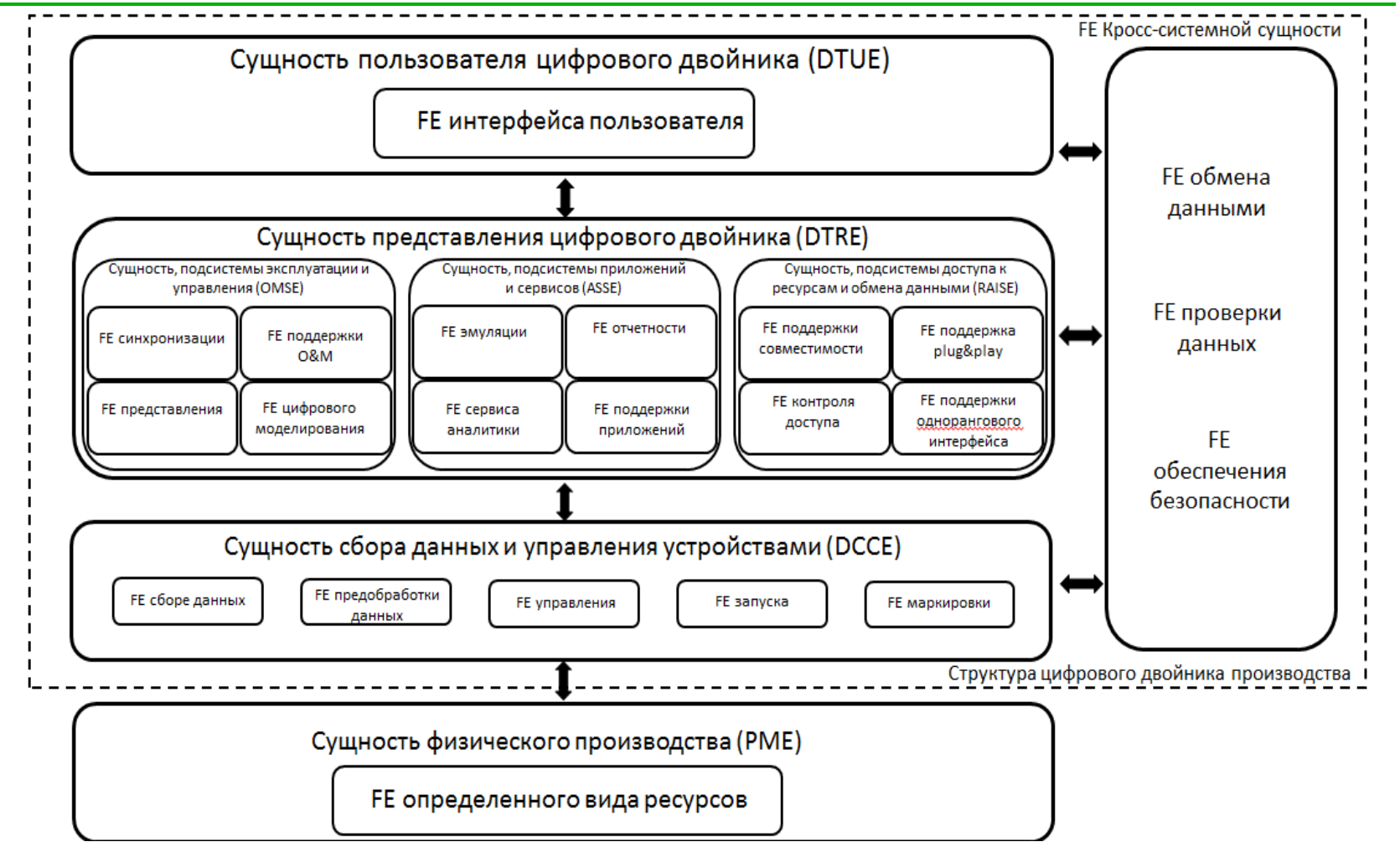

Рис. 1. Функциональное представление типовой архитектуры цифрового двойника производства с декомпозированием на функциональные сущности (FE):

DTUE - Digital Twin User Entity; FE - Functional Entity; DTRE - Digital Twin Representation Entity; OMSE - Operation and Management Sub-system Entity; ASSE - Application and Service Sub-system Entity; RAISE - Resource Access and Interchange Sub-system Entity; O\&M - Operation and Management; PME - Physical Manufacturing Entity

Таблица 1

\section{Функциональная сущность в DCCE}

\begin{tabular}{|l|l|}
\hline \multicolumn{1}{|c|}{ Сущность } & \multicolumn{1}{|c|}{ Задачи } \\
\hline FE сбора данных & $\begin{array}{l}\text { FE сбора данных обеспечивает функционал сбора } \\
\text { данных от РМЕ. }\end{array}$ \\
\hline $\begin{array}{l}\text { FE предобработки } \\
\text { данных }\end{array}$ & $\begin{array}{l}\text { FE предобработки данных обеспечивает функционал } \\
\text { предобработки собранных данных, например } \\
\text { фильтрацию и объединение. }\end{array}$ \\
\hline FE управления & $\begin{array}{l}\text { FE управления обеспечивает функционал управления } \\
\text { РМЕ по запросу DTRE. }\end{array}$ \\
\hline FE запуска & $\begin{array}{l}\text { FE запуска обеспечивает приведение РME в действие по } \\
\text { запросу DTRE аналогично FE управления. }\end{array}$ \\
\hline
\end{tabular}




\begin{tabular}{|l|l|}
\hline \hline FE маркировки & $\begin{array}{l}\text { FE маркировки обеспечивает использование } \\
\text { функционала идентификации РМЕ и его данных, } \\
\text { подлежащих сбору и контролю. }\end{array}$ \\
\hline
\end{tabular}

Функциональная сущность в DTRE (сущность представления цифрового двойника) определяется функциональными сущностями OMSE, ASSE и RAISE, задачи которых приведены соответственно в таблицах 2, 3, 4.

Таблица 2

Функциональная сущность в OMSE

\begin{tabular}{|l|l|}
\hline \multicolumn{1}{|c|}{ Сущность } & \multicolumn{1}{|c|}{ Задачи } \\
\hline FE синхронизации & $\begin{array}{l}\text { FE синхронизации обеспечивает функционал } \\
\text { синхронизации статуса визуализированной цифровой } \\
\text { сущности со статусом наблюдаемого } \\
\text { производственного элемента, или наоборот. }\end{array}$ \\
\hline FE представления & $\begin{array}{l}\text { FE представления вместе с FE цифрового } \\
\text { моделирования обеспечивает функционал } \\
\text { представления наблюдаемого производственного } \\
\text { элемента в виде цифровой сущности. }\end{array}$ \\
\hline FE цифрового & $\begin{array}{l}\text { FЕ цифрового моделирования обеспечивает } \\
\text { функционал интерпретации данных о наблюдаемом } \\
\text { производственном элементе для понимания его } \\
\text { физических свойств, статуса и т д. }\end{array}$ \\
\hline $\begin{array}{l}\text { FE поддержки } \\
\text { О\&M }\end{array}$ & $\begin{array}{l}\text { FЕ поддержки О\&М предоставляет функциональные } \\
\text { возможности для использования и управления DTRE. }\end{array}$ \\
\hline
\end{tabular}

Таблица 3

Функциональная сущность в ASSE

\begin{tabular}{|l|l|}
\hline \multicolumn{1}{|c|}{ Сущность } & \multicolumn{1}{c|}{ Задачи } \\
\hline FE эмуляции & $\begin{array}{l}\text { FЕ эмуляции предоставляет функциональные возможности } \\
\text { для эмуляции. }\end{array}$ \\
\hline $\begin{array}{l}\text { FE сервиса } \\
\text { аналитики }\end{array}$ & $\begin{array}{l}\text { FE сервиса аналитики предоставляет функционал для } \\
\text { анализа данных, полученных от РМЕ, и результата } \\
\text { эмуляции. }\end{array}$ \\
\hline FE отчетности & $\begin{array}{l}\text { FЕ отчетности предоставляет функционал составления } \\
\text { отчета о результатах производства, анализа эмуляции и т.д. }\end{array}$ \\
\hline
\end{tabular}




\begin{tabular}{|l|l|}
\hline FE поддержки & FE поддержки приложений обеспечивает функционал \\
приложений & хостинговой платформы для реализации предиктивных и \\
& $\begin{array}{l}\text { peaктивных, открытых и закрытых приложений (например, } \\
\text { Node.js). }\end{array}$ \\
\hline
\end{tabular}

Таблица 4

Функциональная сущность в RAISE

\begin{tabular}{|c|c|}
\hline Сущность & Задачи \\
\hline $\begin{array}{l}\text { FE обеспечения } \\
\text { совместимости }\end{array}$ & $\begin{array}{l}\text { FE обеспечения совместимости вместе с FE } \\
\text { однорангового интерфейса предоставляют } \\
\text { функционал взаимодействия с другими DTRE. }\end{array}$ \\
\hline $\begin{array}{l}\text { FE контроля } \\
\text { доступа }\end{array}$ & $\begin{array}{l}\text { FE контроля доступа вместе с FE обеспечения } \\
\text { безопасности предоставляют функционал управления } \\
\text { доступом DTUE к PME. }\end{array}$ \\
\hline $\begin{array}{l}\text { FE поддержки } \\
\text { plug\&play }\end{array}$ & $\begin{array}{l}\text { FE поддержки plug\&play вместе с FE поддержки O\&M } \\
\text { обеспечивает функционал динамического } \\
\text { использования РМЕ. т.е. присоединение и выход из } \\
\text { структуры цифрового двойника производства в } \\
\text { процессе выполнения. }\end{array}$ \\
\hline $\begin{array}{l}\text { FE однорангового } \\
\text { интерфейса }\end{array}$ & $\begin{array}{l}\text { FE однорангового интерфейса вместе с FE } \\
\text { обеспечения совместимости обеспечивают } \\
\text { функционал взаимодействия с другими DTRE. }\end{array}$ \\
\hline
\end{tabular}

Функциональная сущность FE пользовательского интерфейса в обеспечивает совместимость DTUE с DTRE.

Функциональные задачи субъекта в кросс-системной сущности (CSE) приведены в таблице 5 .

Таблица 5

Функциональный субъект в CSE

\begin{tabular}{|l|l|}
\hline \multicolumn{1}{|c|}{ Сущность } & \multicolumn{1}{c|}{ Задачи } \\
\hline $\begin{array}{l}\text { FE обмена } \\
\text { данными }\end{array}$ & $\begin{array}{l}\text { FE обмена данными обеспечивает функционал обмена } \\
\text { данными между субъектами структуры цифрового } \\
\text { двойника производства по соответствующим сетевым } \\
\text { протоколам. }\end{array}$ \\
\hline FE проверки & FE проверки данных вместе с FE обеспечения \\
\hline
\end{tabular}




\begin{tabular}{|l|l|}
\hline данных & $\begin{array}{l}\text { безопасности обеспечивают точность и целостность } \\
\text { данных. }\end{array}$ \\
\hline $\begin{array}{l}\text { FЕ обеспечения } \\
\text { безопасности }\end{array}$ & $\begin{array}{l}\text { FЕ обеспечения безопасности обеспечивает } \\
\text { функциональные возможности обеспечения } \\
\text { безопасности структуры цифрового двойника } \\
\text { производства, включая аутентификацию, } \\
\text { авторизацию, конфиденциальность, целостность и т.д. }\end{array}$ \\
\hline
\end{tabular}

Сетевое представление типовой архитектуры определяет сети передачи данных в структуре цифрового двойника производства. Основной задачей сетей передачи данных является обеспечение связи и обмена данными между сущностями в различных доменах.

Для комбинированной типовой модели цифрового двойника производства на основе доменов и сущностей, приведенной в [2, с.7], в сетевом представлении типовой архитектуры рассматриваются три сети передачи данных (рис. 2):

- пользовательская сеть;

- сервисная сеть;

- сеть передачи данных.

Пользовательская сеть. Пользовательская сеть соединяет сущности пользователя цифрового двойника (DTUE) с доменом представления цифрового двойника (DTRD). В том случае, если DTUE является MES (Система управления производством (Manufacturing Execution System)), ERP (Планирование ресурсов предприятия (Enterprise Resource Planning)) или другой системой управления производством или даже структурой другого цифрового двойника производства, этот тип DTUE подключается к RAISE в RAISD по пользовательской сети. Пользовательская сеть использует Интернет и IP. Такие сети могут применять любую из технологий как используемую для обмена данными. 


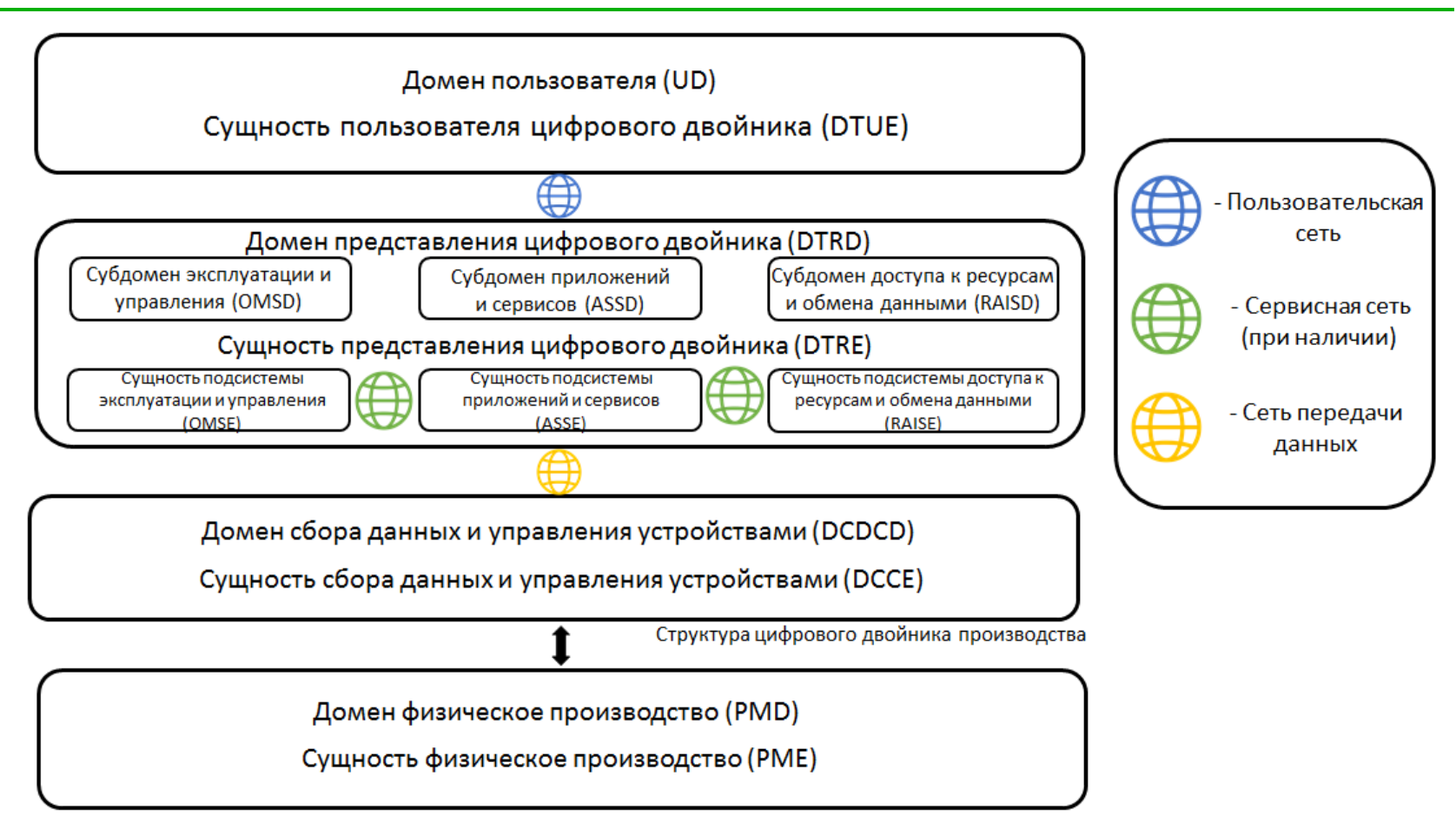

Рис. 2. Сетевое представление типовой архитектуры цифрового двойника производства:

UD - User Domain; DTUE - Digital Twin User Entity; DTRD - Digital Twin Representation Domain; OMSD - Operation and Management Sub-Domain; ASSD - Application and Service Sub-Domain; RAISD - Resource Access and Interchange Sub-Domain; OMSE - Operation and Management Sub-system Entity; ASSE - Application and Service Sub-system Entity; RAISE - Resource Access and Interchange Sub-system Entity; DCDCD - Data Collecting and Device Controlling Domain; DCCE - Data Collecting and Controlling Entity; PMD - Physical Manufacturing Domain; PME - Physical Manufacturing Entity

Сервисная сеть (при наличии). Сервисная сеть соединяет сущности подсистем эксплуатации и управления (OMSE), приложений и сервисов (ASSE) и доступа к ресурсам и обмена данными (RAISE) в субдоменах OMSD, ASSD и RAISD друг с другом соответственно, как правило, посредством проводных сетей, работающих по протоколам на основе IP [3].

Сеть передачи данных. Сеть передачи данных соединяет сущность сбора и управления устройствами (DCCE) в домене сбора и управления устройствами (DCDCD) c доменом представления цифрового двойника (DTRD), т. е. с сущностями субдоменов в OMSD, ASSD и RAISD. Через сеть передачи данных DCCE передает данные, собранные от PME, соответствующим сущностям в OMSD, ASSD и RAISD. Кроме того, информация для управления ресурсами физического производства поступает в 
DCCE от сущностей в OMSD, ASSD и RAISD. Типичные примеры сети передачи данных: локальная проводная сеть, LAN (Local Area Network), беспроводная локальная сеть WLAN (Wireless Local Area Network), и мобильная (сотовая) сеть, которые, как правило, принимают протоколы передачи данных на основе IP независимо от типа сети [2].

Обмен данными между DCCE и DTRE по сети передачи данных обеспечивается соответствующим протоколом передачи данных, например, MTConnect и OPC-UA [4].

Требования к обмену данными по пользовательской сети, по сервисной сети и по сети передачи данных представлены в ПНСТ 432 [4].

Заключение. Функциональное представление архитектуры цифрового двойника промышленного производства основано на логической группировке задач и функций, выполняемых сущностями структуры цифрового двойника и функциональными сущностями. Сущности в домене взаимодействуют с другими сущностями посредством сетей. Сетевое представление типовой архитектуры определяет сети передачи данных в структуре цифрового двойника производства.

\section{Список литературы}

1. ПНСТ 429. Умное производство. Двойники цифровые производства. Часть 1. Общие положения. - М.: Стандартинформ, 2020. -12 с.

2. ПНСТ 430. Умное производство. Двойники цифровые производства. Часть 2. Типовая архитектура. - М.: Стандартинформ, 2020. -16 с.

3. ПНСТ 431. Умное производство. Двойники цифровые производства. Часть 3. Цифровое представление физических производственных элементов. М.: Стандартинформ, 2020. -16 с.

4. ПНСТ 432. Умное производство. Двойники цифровые производства. Часть 4. Обмен информацией. - М.: Стандартинформ, 2020. - 32 с.

(С) Андрианова Л.П., Голубев Д.М., 2021 